\title{
Conditionally Firing Implicative Rules
}

\author{
Martin Štěpnička ${ }^{1}$ Sayantan Mandal ${ }^{2}$ \\ ${ }^{1}$ Institute for Research and Applications of Fuzzy Modeling, CE IT4Innovations, University of Ostrava \\ 30. dubna 22, 70103 Ostrava, Czech Republic \\ martin.stepnicka@osu.cz \\ ${ }^{2}$ Department of Mathematics, Indian Institute of Technology Hyderabad \\ Hyderabad 502 205, India \\ ma10p002@iith.ac.in
}

\begin{abstract}
Conditionally firing rules have been proposed by B. Moser and M. Navara in order to preserve natural properties that are not preserved by the usual setting of fuzzy inference systems such as MamdaniAssilian rules joined to the CRI inference mechanism. In this paper, we follow this direction and show, that if the axioms are naturally modified in order to capture the same semantic for the implicative rules, we can also define conditionally firing implicative rules that jointly with the Bandler-Kohout subproduct preserve exactly the same properties.
\end{abstract}

Keywords: Conditionally firing rules, Compositional Rule of Inference, Bandler-Kohout subproduct, Implicative rules, Fuzzy interpolation

\section{Introduction}

A fuzzy inference system can be seen as a system which derives a meaningful output from an imprecise input. Many kinds of fuzzy inference systems have been studied in the literature $[1,2,3,4]$. In this work, we stem from probably the most favourite ones - from the so called fuzzy relational inference systems - which model a given fuzzy rule base by a single fuzzy relation and the inference mechanism by an appropriate image of a fuzzy set under the fuzzy relation.

\subsection{Fuzzy Rules}

Consider two arbitrary universes $X$ and $Y$. The classes of fuzzy sets on $X$ and $Y$ will be denoted by $\mathcal{F}(X)$ and $\mathcal{F}(Y)$, respectively. The information present in a given fuzzy rule base is contained in pairs of input-output fuzzy sets $\left(A_{1}, B_{1}\right), \ldots$, $\left(A_{n}, B_{n}\right)$, expressing that fuzzy set $B_{i} \in \mathcal{F}(Y)$ is assigned to fuzzy set $A_{i} \in \mathcal{F}(X)$ [5].

There exist two standard approaches to model a given fuzzy rule base by an appropriate fuzzy relation $R \in \mathcal{F}(X \times Y)$.

The first approach consists in constructing the fuzzy relation $\hat{R} \in \mathcal{F}(X \times Y)$ defined by

$$
\hat{R}(x, y)=\bigwedge_{i=1}^{n}\left(A_{i}(x) \rightarrow B_{i}(y)\right)
$$

where $\rightarrow$ is a fuzzy implication [6]. As stated by Dubois et al. [7]: "In the above view, each piece of information (fuzzy rule) is viewed as a constraint. This view naturally leads to a conjunctive way of merging the individual pieces of information since the more information, the more constraints and the less possible values to satisfy them." This fact together with the fact that the minimum operation (as well as other t-norms) is an appropriate interpretation of conjunction (the logical operation AND) and residual operation is an appropriate interpretation of implication (the logical operation IF-THEN), the above statement leads to the conclusion that the fuzzy relation $\hat{R}$ defined by (1) is a proper model of the following set of fuzzy rules

$$
\begin{array}{ccc}
\text { IF } x \text { is } \mathcal{A}_{1} & \text { THEN } & y \text { is } \mathcal{B}_{1} \\
& \text { AND } \\
& \ldots & \\
\text { IF } x \text { is } \mathcal{A}_{n} & \text { THEN } & y \text { is } \mathcal{B}_{n}
\end{array}
$$

where $\mathcal{A}_{i}$ and $\mathcal{B}_{i}$ are predicates represented by fuzzy sets $A_{i} \in \mathcal{F}(X)$ and $B_{i} \in \mathcal{F}(Y)$.

The second approach to modelling a given fuzzy rule base, initiated by a successful experimental application by Mamdani and Assilian [8], consists in constructing the fuzzy relation $\check{R} \in \mathcal{F}(X \times Y)$ defined by

$$
\check{R}(x, y)=\bigvee_{i=1}^{n}\left(A_{i}(x) * B_{i}(y)\right)
$$

where $*$ is a t-norm. Obviously, the fuzzy relation $\check{R}$ can hardly be considered as a model of fuzzy rule base (2). As mentioned above, a t-norm is an appropriate interpretation of conjunction, not of implication; moreover, the maximum operation disjunctively aggregating all rules has nothing in common with the logical operation AND.

We again recall the work of Dubois et al. [7]: "It seems that fuzzy rules modelled by (3) are not viewed as constraints but are considered as pieces of data. Then the maximum in (3) expresses accumulation of data." This fact together with the known fact that the maximum operation as well as other t-conorms are appropriate interpretations of disjunction (the logical connective OR) $[9,10]$ leads 
to the conclusion that the fuzzy relation $\check{R}$ defined by (3) is a proper model of the following set of fuzzy rules

$$
\begin{array}{ccc}
x \text { is } \mathcal{A}_{1} & \text { AND } & y \text { is } \mathcal{B}_{1} \\
& \ldots & \\
& \text { OR } & \\
& \ldots & \\
x \text { is } \mathcal{A}_{n} & \text { AND } & y \text { is } \mathcal{B}_{n}
\end{array}
$$

It is worth mentioning that distinguishing between the conditional (IF-THEN) form of fuzzy rules (2) and the Cartesian product (AND) form of fuzzy rules (4) at the syntactical level is not commonly done, but it can be found e.g. in [11, 12]. Usually only the form (2) is considered because of several, mainly historical, reasons and the differences are taken into account only at the semantical level. But the differences can play a crucial role in further implementations and, therefore, they should be kept in mind. For the rest of this paper, the distinction between both types of rules and their semantics is also crucial. For a detailed discussion on both forms of fuzzy rules, we refer to the relevant literature $[7,9,10,13]$.

\subsection{Inference mechanisms}

There are many inference mechanisms that with help of fuzzy rules (or their models) deduce an appropriate output $B^{\prime} \in \mathcal{F}(Y)$ based on a given observation (input) $A^{\prime} \in \mathcal{F}(X)$. In this investigation, we concentrate only on fuzzy relational inference systems that directly use a fuzzy relation $R \in \mathcal{F}(X \times Y)$ as a model of fuzzy rules, and an image of a fuzzy set under the fuzzy relation as a model of the inference mechanism. In most cases, one uses the direct image (sup-* composition),

$$
B^{\prime}=A^{\prime} \circ R,
$$

also called Compositional Rule of Inference (CRI) [2] which is defined by

$$
\left(A^{\prime} \circ R\right)(y)=\bigvee_{x \in X}\left(A^{\prime}(x) * R(x, y)\right) .
$$

Besides the CRI, another alternative we may use is the subdirect image (inf- $\rightarrow$ composition)

$$
B^{\prime}=A^{\prime} \triangleleft R
$$

which is related to the Bandler-Kohout subproduct (BK-subproduct) [14]. It is defined by

$$
\left(A^{\prime} \triangleleft R\right)(y)=\bigwedge_{x \in X}\left(A^{\prime}(x) \rightarrow R(x, y)\right) .
$$

The BK-subproduct was firstly suggested as an inference mechanism in [15] and later on, in [16], it was shown that both inference mechanisms are equally good. Particularly, the advantages, that may be obtained from using one or the other inference mechanism, do not come from the mechanism itself, but from a proper combination of the mechanism and an appropriate choice of a model of fuzzy rules. If an inference mechanism provides some advantage when connected with model $\check{R}$, the same holds for the other inference mechanisms and the model $\hat{R}$ and vice-versa, the same holds about disadvantages. There is no advantage that would be preserved for any of the two above mentioned inferences that would hold generally no matter the choice of the fuzzy rule base model, see [16, 17].

\subsection{Mathematical Background}

For the rest of the paper, let us fix a complete residuated lattice

$$
\langle[0,1], \wedge, \vee, *, \rightarrow 0,1\rangle
$$

as the background algebraic structure [10]. In other words, the multiplicative operation $*$ is a leftcontinuous t-norm and correspondingly the fuzzy implication $\rightarrow$ is a residual implication and they both form an adjoint pair. Furthermore, we define the fuzzy equivallence in a standard way, i.e., $a \leftrightarrow b=(a \rightarrow b) \wedge(b \rightarrow a)$. Specifically, wherever we consider $\check{R}$ or $\hat{R}$, the connectives involved in them are from residuated lattice structure. Note, that one can find interesting works where the chosen operations do not form a residuated lattice, see $[18,19,20]$.

\subsection{Motivation}

From the interpolation point of view, BKsubproduct is preferred when dealing with $\hat{R}$ while CRI should be preferred for conjunctive rules $\check{R}$. On the other hand, the most usual case is opposite, particularly, most usually the CRI is combined with the Mamdani rules. The reasons are historical (both CRI and $\check{R}$ were applied and thus, spread among practitioners, much earlier and therefore, they became a sort of state-of-art or the "first choice" for them) as well as practical (robustness and also equivalence of FITA and FATI and existence of equivalent hierarchical inference structure and thus, lower computational computational efforts, see $[16,17])$.

However, as it may be shown, this combination, though generally accepted and bringing many advantages, is yet also very problematic. For example, for many operations modeling the conjunction, particularly, for t-norms with zero divisors, it is impossible to construct an intuitive and natural model of fuzzy rules such that it ensures fuzzy interpolativity as well as meaningful conclusions [21] or reaching extreme output values after an appropriate defuzzification. B. Moser and M. Navara approached these problems and suggested for the combination of $\check{R}$ and CRI the so called conditionally firing rules, i.e., a CRI-modified inference system that focuses on the elimination of both mentioned problems, see also $[22]$. 
The problems mentioned above can be shown to be present also for the combination of BKsubproduct and $\hat{R}$. In a similar fashion for this combination of $\triangleleft$ and $\hat{R}$, conditionally firing implicative rules can be proposed which forged our motivation to propose and investigate conditionally firing implicative rules.

\section{Fuzzy Interpolation}

Each inference mechanism should posses a fundamental property - preservation of modus ponens. In other words, if an input fuzzy set $A^{\prime} \in \mathcal{F}(X)$ is equal to one of the consequents, say $A_{i}$, then the inferred output $B^{\prime} \in \mathcal{F}(Y)$ should be equal to the respective consequent $B_{i}$. This requirement leads to the following systems of fuzzy relational equations

$$
A_{i} @ R=B_{i}, \quad i=1, \ldots, n
$$

where @ $\in\{0, \triangleleft\}$ and $R$ is an unknown fuzzy relation on $X \times Y$. A fuzzy relation $R \in \mathcal{F}(X \times Y)$ which satisfies (9) is called a solution of the system and we say, that $R$ interpolates $\left(A_{i}, B_{i}\right)$. In such a case, $R$ be seen as a correct model of the given fuzzy rule base in the given fuzzy inference system.

\subsection{State-of-Art in Fuzzy Interpolation}

Obviously, not all systems (9) are solvable, i.e., not for all sets of pairs $\left(A_{i}, B_{i}\right)_{i=1}^{n}$ there exists a fuzzy relations that would interpolate them. The question of solvability of such systems was addressed by many researchers and in this Section, we recall only the most fundamental results $[23,24,25,26]$.

Theorem 1 System (9) with @ = $0(@=\triangleleft)$ is solvable if and only if $\hat{R}(\check{R})$ is a solution of this system. In case of solvability, $\hat{R}(\check{R})$ is the greatest (least) solution of $(9)$ with $@=\circ(@=\triangleleft)$.

Theorem 1 actually states that the implicative model $\hat{R}$ (or Mamdani-Assilian model $\check{R}$ ) should be the first choice whenever dealing with the inference mechanism modelled by $\circ$ (or $\triangleleft$, respectively). If there exist some reasons, for example preservation of robustness [16], computational complexity [16] or the existence of hierarchical inference that is identical to the non-hierarchical one $[16,27]$, why the combination of Mamdani-Assilian model $\check{R}$ and the CRI inference $\circ$ (implicative model $\hat{R}$ and the BKsubproduct inference $\triangleleft$ ) should be preferred, one should first of all check whether the interpolativity is preserved also in this case. The answer to this question is provided by the following theorems that collects results from $[25,28,29]$.

Theorem 2 Let all $A_{i}, i=1, \ldots, n$, be normal. Then $\check{R}(\hat{R})$ is a solution of $(9)$ with $@=\circ(@=\triangleleft)$ if and only if the condition

$$
\bigvee_{x \in X}\left(A_{i}(x) * A_{j}(x)\right) \leq \bigwedge_{y \in Y}\left(B_{i}(y) \leftrightarrow B_{j}(y)\right)
$$

holds for any $i, j \in\{1, \ldots, n\}$.

Note that condition (10) may be very restrictive, as shown in [21] and discussed below.

\subsection{Conditionally Firing Rules}

Let us recall, that B. Moser and M. Navara [21] introduced the following three axioms for MamdaniAssilian rules and CRI inference.

Axiom1) For all $i \in\{1, \ldots, n\}$

$$
A_{i} \circ \check{R}=B_{i} \text {; }
$$

Axiom2) For each normal input $A^{\prime} \in \mathcal{F}(X)$ there is an index $i$ such that

$$
A^{\prime} \circ \check{R} \nsubseteq B_{i}
$$

Axiom3) The output $A^{\prime} \circ \check{R}$ belongs to the union of consequents $B_{i}$ of "fired rules", i.e.,

$$
A^{\prime} \circ \check{R} \subseteq \bigcup_{i \in F} B_{i}
$$

where $F=\left\{i \mid \operatorname{Supp}\left(A_{i}\right) \cap \operatorname{Supp}\left(A^{\prime}\right) \neq \emptyset\right\}$ and $\left(B_{i} \cup B_{j}\right)(y)=B_{i}(y) \vee B_{j}(y)$.

Axiom1) is the already discussed fuzzy interpolation property.

Axiom2), according to [21], ensures the significance of each output set. It implies that for each normal input the inference mechanism provides a significant output giving non-trivial information.

Finally, Axiom3) is a weak monotonicity of outputs provided that the rules are locally monotonic, i.e., in some interval, the rules describe a monotonic relation [21]. Note that originally, Axiom3) was a bit different, it assumed that the inferred output is contained in the convex hull of consequents of fired rules. However, the authors actually proved that the Axiom3) from this article is preserved for the Mamdani-Assilian systems, and the fact, that it holds also for the convex hull, is just a direct consequent, see [21].

Let us recall the main results from [21].

Proposition 1 [21] Let $*$ be a t-norm without zero divisors. Let all $A_{i}, i=1, \ldots, n$, be continuous and normal and let $B_{i}, i=1, \ldots, n$, be fuzzy sets with mutually different supports. Then the MamdaniAssilian model $\check{R}$ and CRI inference o do not satisfy Axiom1) and Axiom2) simultaneously.

Proposition 2 [21] The Mamdani-Assilian model $\check{R}$ and CRI inference o violate Axiom2) iff there is an $x^{\prime} \in X$ such that $\forall i \in\{1, \ldots, n\}$

$$
A_{i}\left(x^{\prime}\right) \leq \bigwedge_{j=1}^{n} \bigwedge_{y \in Y}\left(B_{i}(y) \rightarrow B_{j}(y)\right)
$$


In other words, Mamdani-Assilian with CRI is not the best choice as it either requires a use of a tnorm with zero divisors or, it does not allow to preserve all three axioms simultaneously, apart from very unintuitive settings such as equal supports of all consequents fuzzy sets.

Therefore, B. Moser and M. Navara proposed the conditionally firing rules which enables to combine the Mamdani-Assilian model $\check{R}$ and CRI in order to satisfy Axiom1)-Axiom3) as follows:

$$
\left(A^{\prime} \circ_{c} \check{R}\right)(y)=\sigma^{[-1]}\left(\bigvee_{i=1}^{n}\left(C_{i}\left(A^{\prime}\right) * \sigma\left(B_{i}(y)\right)\right)\right)
$$

where $C_{i}\left(A^{\prime}\right)$ is the degree of conditional firing of the $i$-th rule and is defined as follows:

$$
C_{i}\left(A^{\prime}\right)=\frac{\bigvee_{x \in X} \rho\left(A^{\prime}(x)\right) * \rho\left(A_{i}(x)\right)}{\bigvee_{j=1}^{n}\left(\bigvee_{x \in X} \rho\left(A^{\prime}(x)\right) * \rho\left(A_{j}(x)\right)\right)} .
$$

Here $\rho:[0,1] \rightarrow[0,1]$ and $\sigma:[0,1] \rightarrow[c, 1]$ with $0 \leq$ $c<1$ are increasing bijections with pseudoinverse $\sigma^{[-1]}$ defined as follows

$$
\sigma^{[-1]}(t)=\left\{\begin{array}{l}
\sigma^{-1}(t), \quad \text { if } t \geq c \\
0, \quad \text { otherwise. }
\end{array}\right.
$$

Unlike in the standard firing, this degree of conditional firing $C_{i}\left(A^{\prime}\right)$ depends on all antecedent fuzzy sets $A_{j}$ and, moreover, for a normal input $A^{\prime}$, at least one of values $C_{i}\left(A^{\prime}\right)$ equals to one.

Theorem 3 [21] Let $\left(A_{i}, B_{i}\right)_{i=1}^{n}$ be a fuzzy rule base, let $A_{i}$ be normal, let $\rho:[0,1] \rightarrow[0,1]$ be any automorphism satisfying the conditions:

Con1) "covering antecedents":

$$
\bigwedge_{x \in X} \bigvee_{i=1}^{n} A_{i}(x)>0
$$

Con2) "disjointness of antecedents": $\exists c<1$ s.t.

$$
\bigvee_{x \in X} \rho\left(A_{i}(x)\right) * \rho\left(A_{j}(x)\right) \leq c, \quad \text { whenever } i \neq j
$$

Con3) "significance of consequents": for each $i \in$ $\{1, \ldots, n\}$, there is a $y_{i} \in Y$ satisfying

$$
B_{i}\left(y_{i}\right)>\bigwedge_{i \neq j} B_{j}\left(y_{i}\right) .
$$

Then for any isomorphism $\sigma:[0,1] \rightarrow[c, 1]$ the mapping (12) satisfies axioms Axiom1)-Axiom3).

In other words, under very mild assumptions Con1)-Con3), the CRI inference based on conditionally firing Mamdani-Assilian rules satisfies Axiom1)-Axiom3) simultaneously, which is not the case of standard CRI and $\check{R}$ without any restriction on the used t-norm, see Proposition 1.

Moreover, the following Proposition confirms a desirable behavior of the suggested system if one of the rules is fired in the highest degree.
Proposition 3 [21] Under the assumptions Con1)Con3) and for $A^{\prime}$ such that $A^{\prime}=\chi_{x^{\prime}}$ for some $x^{\prime} \in X$ such that $A_{i}\left(x^{\prime}\right)=1$ for some fixed $i \in$ $\{1, \ldots, n\}$. Then

$$
A^{\prime} \circ_{c} \check{R}=B_{i} .
$$

\section{Conditionally Firing Implicative Rules}

In this Section, we suitably modify the axioms Axiom1)-Axiom3) so that they can express the same what they expressed in the case of MamdaniAssilian rules.

\subsection{Modified Axioms}

Let us unfold the meanings of Axiom1)-Axiom3). Axiom1) - the interpolativity - is unquestionable and has to be preserved by any inference system. Thus, also by the system with implicative rules and the BK-subproduct.

However, in the case of implicative rules, Axiom2)-Axiom3) no longer express the same what they expressed in the case of Mamdani-Assilian rules. Axiom2) expressed a sort of significance of outputs in case of normal inputs. It was motivated by the fact that trivial outputs are empty. But in the case of implicative rules, trivial outputs are those that are equal to one on the whole output universe. The more significant outputs, the lower the membership degrees of output fuzzy sets, which is a consequence of the logical constraint nature of the implicative rules.

Similar situation relates to Axiom3). If a conjunctive rule is fired in a certain degree, the inferred output is nothing else but a cartesian product of the respective consequent and the firing degree, which is, obviously, a fuzzy set that is a "subset" of the consequent. If more rules are fired, the subsethood is preserved w.r.t. to the union of all such consequents. However, in case of the implicative model, again, the opposite assumption is natural. If an implicative rule, is fired, the inferred output is a fuzzy set obtained as respective consequent implied the firing degree. This fuzzy set is then necessarily a "superset" of the consequent. And again, for the case of more fired rules, with having in mind that these rules are conjunctively aggregated by a minimum, the inferred output is greater or equal to the intersection of all fired consequents.

Therefore, we introduce the modified axioms as follows:

Axiom1') For all $i \in\{1, \ldots, n\}$

$$
A_{i} \triangleleft \hat{R}=B_{i} ;
$$

Axiom2') For each normal input $A^{\prime} \in \mathcal{F}(X)$, there is an index $i$ such that

$$
A^{\prime} \triangleleft \hat{R} \nsupseteq B_{i} ;
$$


Axiom3') The output $A^{\prime} \triangleleft \hat{R}$ contains the intersection of consequents $B_{i}$ of "fired rules", i.e.,

$$
A^{\prime} \triangleleft \hat{R} \supseteq \bigcap_{i \in F} B_{i}
$$

where $F=\left\{i \mid \operatorname{Supp}\left(A_{i}\right) \cap \operatorname{Supp}\left(A^{\prime}\right) \neq \emptyset\right\}$ and $\left(B_{i} \cap B_{j}\right)(y)=B_{i}(y) \wedge B_{j}(y)$.

We argue, that for the combination of the BKsubproduct $\triangleleft$ and the implicative model $\hat{R}$, the axioms Axiom1')-Axiom3') express exactly the same desirable properties as those introduced by B. Moser and M. Navara in [21] and [22].

Now, for the implicative rules and with the modified axiomatization, let us consider the same questions that have been addressed in [21].

Proposition 4 Let $*$ be a left-continuous t-norm without zero divisors. Let $A_{i}, i=1 \ldots, n$ be continuous and normal and let $B_{i}, i=1, \ldots, n$ be fuzzy sets with mutually different supports. Then the implicative model $\hat{R}$ and BK-subproduct $\triangleleft$ do not satisfy Axiom1') and Axiom2') simultaneously.

Sketch of the proof: The proof is analogous to the one of Proposition 1. For any $i \neq j$

$$
\bigwedge_{y \in Y}\left(B_{i}(y) \leftrightarrow B_{j}(y)\right)=0
$$

and thus, the following also needs to hold

$$
\bigvee_{x \in X}\left(A_{i}(x) * A_{j}(x)\right)=0 \text {. }
$$

For a t-norm without zero divisors, the latter is fulfilled only if the supports of antecedents $A_{i}$ are disjoint. Let us consider $x^{\prime} \notin \operatorname{Supp}\left(A_{i}\right)$ for any $i$ and the respective singleton $A^{\prime}=\chi_{x^{\prime}}$.

Then the proof continuous by showing that for any $y \in Y$, the inferred output $\left(A^{\prime} \triangleleft \hat{R}\right)(y)$ is equal to 1 and thus,

$$
A^{\prime} \triangleleft \hat{R} \supseteq B_{i}
$$

for any $i$ which violates Axiom2').

Proposition 5 The implicative model $\hat{R}$ and $B K$ subproduct $\triangleleft$ violate Axiom2') iff there is an $x^{\prime} \in X$ such that

$\forall i \in\{1, \ldots, n\}: A_{i}\left(x^{\prime}\right) \leq \bigwedge_{j=1}^{n} \bigwedge_{y \in Y}\left(B_{i}(y) \rightarrow B_{j}(y)\right)$.

Sketch of the proof: Axiom2') is violated if $A^{\prime} \triangleleft$ $\hat{R} \supseteq B_{j}$ for all indexes $j$, which occurs if and only if $\forall j, \forall y$

$$
\begin{aligned}
& B_{j}(y) \leq \bigwedge_{x \in X}\left(A^{\prime}(x) \rightarrow \bigwedge_{i=1}^{n}\left(A_{i}(x) \rightarrow B_{i}(y)\right)\right) \\
\Longleftrightarrow & B_{j}(y) \leq A^{\prime}(x) \rightarrow \bigwedge_{i=1}^{n}\left(A_{i}(x) \rightarrow B_{i}(y)\right) \quad \forall x .
\end{aligned}
$$

If the above holds for all $x \in X$, it has to hold also for a particular $x^{\prime}$ for which $A^{\prime}\left(x^{\prime}\right)=1$ which after a double application of the adjunction property proves the Proposition.
Proposition 6 The implicative model $\hat{R}$ and $B K$ subproduct $\triangleleft$ satisfy Axiom3').

Sketch of the proof: For arbitrary $y$ the inferred output

$$
\begin{aligned}
\left(A^{\prime} \triangleleft \hat{R}\right)(y) & \\
= & \bigwedge_{x \in X}\left(A^{\prime}(x) \rightarrow \bigwedge_{i=1}^{n}\left(A_{i}(x) \rightarrow B_{i}(y)\right)\right) \\
= & \bigwedge_{x \in X}\left(A^{\prime}(x) \rightarrow \bigwedge_{i \notin F}\left(A_{i}(x) \rightarrow B_{i}(y)\right)\right. \\
& \left.\wedge \bigwedge_{i \in F}\left(A_{i}(x) \rightarrow B_{i}(y)\right)\right)
\end{aligned}
$$

which after some steps leads to

$$
\begin{aligned}
& =\bigwedge_{i \in F}\left(\bigvee_{x \in X}\left(A^{\prime}(x) * A_{i}(x)\right) \rightarrow B_{i}(y)\right) \\
& \geq \bigwedge_{i \in F} B_{i}(y)
\end{aligned}
$$

which proves that $A^{\prime} \triangleleft \hat{R} \supseteq \bigcap_{i \in F} B_{i}$.

\subsection{Conditionally Firing Implicative Rules with BK-subproduct}

We adopt the same assumptions for $\rho, \sigma, \sigma^{[-1]}$ and $c$ that were recalled in Section 2.2 for the MamdaniAssilian rules. Furthermore, we keep the same definition of degree of conditional firing of the $i$-th rule given by (13).

The given setting allows us to define the conditionally firing implicative rules inference based on the BK-subproduct as follows:

$$
\left(A^{\prime} \triangleleft_{c} \hat{R}\right)(y)=\sigma^{[-1]}\left(\bigwedge_{i=1}^{n}\left(C_{i}\left(A^{\prime}\right) \rightarrow \sigma\left(B_{i}(y)\right)\right)\right) .
$$

Let us note, that condition Con3) from Theorem 3 was again designed for the purpose of Mamdani-Assilian rules. Indeed, in this type of conjunctive rules, the significance is viewed as significantly "high" or by the other consequents "uncovered". However, in case of implicative rules, the insignificant constraint is provided by such a rule that by its consequent encompass all the others. Therefore, we again modify the original conditions Con1)Con3) in such a way that the modified one express the same idea for implicative rules. The only condition that needs to be modified is Con3). However, in order to avoid misunderstandings, we denote all three conditions by Con1')-Con3') although Con1') and Con2') are equivalent to the original Con1) and Con2), respectively.

Then we can introduce the following Theorem. 
Theorem 4 Let $\left(A_{i}, B_{i}\right)_{i=1}^{n}$ be a fuzzy rule base, let $A_{i}$ be normal, let $\rho:[0,1] \rightarrow[0,1]$ be any automorphism satisfying the conditions

Con1') "covering antecedents":

$$
\bigwedge_{x \in X} \bigvee_{i=1}^{n} A_{i}(x)>0
$$

Con2') "disjointness of antecedents": $\exists c<1$ s.t.

$$
\bigvee_{x \in X} \rho\left(A_{i}(x)\right) * \rho\left(A_{j}(x)\right) \leq c, \quad \text { whenever } i \neq j
$$

Con3') "significance of consequents": for each $i \in\{1, \ldots, n\}$, there is a $y_{i} \in Y$ satisfying

$$
B_{i}\left(y_{i}\right)<\bigvee_{i \neq j} B_{j}\left(y_{i}\right)
$$

Then for any isomorphism $\sigma:[0,1] \rightarrow[c, 1]$ the mapping (16) satisfies axioms Axiom1')-Axiom3').

Sketch of the proof: Since all antecedents are normal, the denominator in (13) always equals to one and thus,

$$
C_{j}\left(A_{i}\right)=\bigvee_{x \in X} \rho\left(A_{i}(x)\right) * \rho\left(A_{j}(x)\right)
$$

and the corresponding output is given

$$
\left(A_{i} \triangleleft_{c} \hat{R}\right)(y)=\sigma^{[-1]}\left(\bigwedge_{j=1}^{n}\left(C_{j}\left(A_{i}\right) \rightarrow \sigma\left(B_{j}(y)\right)\right)\right) .
$$

For $j \neq i$ Con2') implies

$$
C_{j}\left(A_{i}\right) \rightarrow \sigma\left(B_{j}(y)\right) \geq c \rightarrow \sigma\left(B_{j}(y)\right)=1
$$

and thus

$$
\begin{aligned}
\left(A_{i} \triangleleft_{c} \hat{R}\right)(y) & =\sigma^{[-1]}\left(C_{i}\left(A_{i}\right) \rightarrow \sigma\left(B_{i}(y)\right)\right) \\
& =\sigma^{[-1]}\left(1 \rightarrow \sigma\left(B_{i}(y)\right)\right) \\
& =\sigma^{[-1]}\left(\sigma\left(B_{i}(y)\right)\right)=B_{i}(y)
\end{aligned}
$$

which proves the preservation of Axiom1').

In order to prove Axiom2'), it is sufficient to consider the "worst" case when the input fuzzy set $A^{\prime}$ is a singleton, i.e., $A^{\prime}=\chi_{x^{\prime}}$ for some $x^{\prime} \in X$. According to Con1'), at least one rule fires totally, i.e., $C_{i}\left(A^{\prime}\right)=1$ for some $i$. Then it is possible to show that $\left(A^{\prime} \triangleleft_{c} \hat{R}\right)(y)=B_{i}(y)$ which together with Con3') implies Axiom2').

Preservation of Axiom3') is proved analogously as in the proof of Proposition 6.

Moreover, similarly to [21], we get the following Proposition describing a desirable behavior when one rule fires totally.

Proposition 7 Let us make the same assumption as in Theorem 4. Let $A^{\prime}=\chi_{x^{\prime}}$ for some $x^{\prime} \in X$ such that $A_{i}\left(x^{\prime}\right)=1$ for some fixed $i$. Then

$$
A^{\prime} \triangleleft_{c} \hat{R}=B_{i} .
$$

Sketch of the proof:

It is possible to show that the degrees of conditionally firing rules are $C_{i}\left(A^{\prime}\right)=1$ and $C_{j}\left(A^{\prime}\right) \leq c$ for $j \neq i$. We obtain for all $y \in Y$

$$
\begin{aligned}
\sigma^{[-1]} & \left(C_{i}\left(A^{\prime}\right) \rightarrow \sigma\left(B_{i}(y)\right)\right) \\
& =\sigma^{[-1]}\left(1 \rightarrow \sigma\left(B_{i}(y)\right)\right)=B_{i}(y),
\end{aligned}
$$

and for $j \neq i$

$$
\begin{aligned}
\sigma^{[-1]} & \left(C_{j}\left(A^{\prime}\right) \rightarrow \sigma\left(B_{j}(y)\right)\right) \\
& \geq \sigma^{[-1]}\left(c \rightarrow \sigma\left(B_{j}(y)\right)\right) \\
& =\sigma^{[-1]}(1)=1
\end{aligned}
$$

because $c \leq \sigma\left(B_{j}(y)\right)$ for any $y$, and as the minimum of these fuzzy sets, $A^{\prime} \triangleleft_{c} \hat{R}=B_{i}$ which proves the Proposition.

\section{Concluding Remarks}

The motivation for this paper comes from the fact that very natural properties/axioms expected from fuzzy inference systems are not often preserved in case of the use of standard settings, e.g. in case of the use of Mamdani-Assilian rules and the CRI inference. This fact motivated B. Moser and M. Navara to introduce conditionally firing rules of the Mamdani-Assilian type that jointly with the CRI based inference mechanism preserve the natural axioms under very mild assumptions [21].

The authors also showed that similarly to the case of conjunctive rule, for the implicative one, the given axioms are not preserved either. However, this "failure" of the implicative rules is a direct consequence of the fact that the provided axiomatization was specifically determined in order to capture the natural properties for the case of conjunctive (Mamdani-Assilian) rules. In this paper, we modify the axioms from [21] in such a way, that they capture precisely the same properties as the original ones, but they are specifically designed for the implicative rules. Then we define the conditionally firing implicative rules with the BK-subproduct based inference mechanism. Analogously to the work of Moser and Navara, we show that even these rules preserve the given axioms under very mild conditions.

\section{Acknowledgements}

This work was supported by the European Regional Development Fund in the IT4Innovations Centre of Excellence project (CZ.1.05/1.1.00/02.0070).

\section{References}

[1] D. Driankov, H. Hellendoorn, and M. Reinfrank. An introduction to fuzzy control (2nd ed.). Springer-Verlag, London, UK, 1996. 
[2] L. A. Zadeh. Outline of a new approach to the analysis of complex systems and decision processes. IEEE Trans. Syst. Man Cybern., 3(1):28-44, 1973.

[3] W. Bandler and L. J. Kohout. Fuzzy relational products and fuzzy implication operators. In Proc. Int. Workshop on Fuzzy Reasoning Theory and Applications, London, 1978. Queen Mary College.

[4] P. Magrez and P. Smets. Fuzzy modus ponens: A new model suitable for applications in knowledge-based systems. International Journal of Intelligent Systems, 4(2):181-200, 1989.

[5] T. Vetterlein and M. Štěpnička. Completing fuzzy if-then rule bases by means of smoothing splines. International Journal of Uncertainty, Fuzziness and Knowledge-Based Systems, 14:235-244, 2006.

[6] M. Baczyński and B. Jayaram. Fuzzy Implications. Springer-Verlag, Heidelberg, 2008.

[7] D. Dubois and H. Prade. What are fuzzy rules and how to use them. Fuzzy Sets and Systems, 84:169-185, 1996.

[8] E. H. Mamdani and S. Assilian. An experiment in linguistic synthesis with a fuzzy logic controller. Int. J. Man-Mach. Stud., 7:1-13, 1975.

[9] P. Hájek. Metamathematics of Fuzzy Logic, volume 4 of Trends in Logic. Kluwer Academic Publishers, Dordrecht, 1998.

[10] V. Novák, I. Perfilieva, and J. Močkoř. Mathematical Principles of Fuzzy Logic. Kluwer Academic Publishers, Boston, 1999.

[11] M. Daňková. On approximate reasoning with graded rules. Fuzzy Sets and Systems, 158:652673, 2007.

[12] V. Novák and S. Lehmke. Logical structure of fuzzy IF-THEN rules. Fuzzy Sets and Systems, 157(15):2003-2029, 2006.

[13] F. Klawonn and V. Novák. The relation between inference and interpolation in the framework of fuzzy systems. Fuzzy Sets and Systems, 81(3):331-354, 1996.

[14] W. Bandler and L. J. Kohout. Fuzzy relational products as a tool for analysis and synthesis of the behaviour of complex natural and artificial systems. In S. K. Wang and P. P. Chang, editors, Fuzzy Sets: Theory and Application to Policy Analysis and Information Systems, pages 341-367. Plenum Press, New York, 1980.

[15] W. Pedrycz. Applications of fuzzy relational equations for methods of reasoning in presence of fuzzy data. Fuzzy Sets and Systems, 16:163$175,1985$.

[16] M. Štěpnička and B. Jayaram. On the suitability of the Bandler-Kohout subproduct as an inference mechanism. IEEE Transactions on Fuzzy Systems, 18(2):285-298, 2010.

[17] M. Štěpnička and B. Jayaram. On the computational aspects of the BK-subproduct infer- ence mechanism. In Proc. International Conference on Fuzzy Systems (FUZZ-IEEE'09), pages 1181-1186, Jeju, Korea, 2009.

[18] M. F. Kawaguchi and M. Miyakoshi. Composite fuzzy relational equations with noncommutative conjunctions. Inf. Sci. Inf. Comput. Sci., 110:113-125, 1998.

[19] S. Mandal and B. Jayaram. Bandler-Kohout subproduct with Yager's classes of fuzzy implications. IEEE Transactions on Fuzzy Systems, 22(3):469-482, 2014.

[20] S. Mandal and B. Jayaram. Bandler-Kohout subproduct with Yager's classes of fuzzy implications. IEEE Transactions on Fuzzy Systems, 22:469-482, 2014.

[21] B. Moser and M. Navara. Fuzzy controllers with conditionally firing rules. IEEE Trans. Fuzzy Systems, 10(3):340-348, 2002.

[22] M. Navara and M. Petrík. How to use controller with conditionally firing rules. In M. Štěpnička, V. Novák, and U. Bodenhofer, editors, New Dimensions in Fuzzy Logic and Related Technologies. Proc. 5th EUSFLAT Conference, volume 2, pages 87-94, Ostrava, 2007.

[23] B. De Baets. Analytical solution methods for fuzzy relational equations. In D. Dubois and H. Prade, editors, The Handbook of Fuzzy Set Series Vol. 1, pages 291-340. Academic Kluwer Publ., Boston, 2000.

[24] G.J. Klir and B. Yuan. Fuzzy Sets and Fuzzy Logic. Prentice Hall, New Jersey, 1995.

[25] F. Klawonn. Fuzzy points, fuzzy relations and fuzzy functions. In V. Novák and I. Perfilieva, editors, Discovering the World with Fuzzy Logic, pages 431-453. Springer, Berlin, 2000.

[26] A. Di Nola, S. Sessa, W. Pedrycz, and E. Sanchez. Fuzzy Relation Equations and Their Applications to Knowledge Engineering. Kluwer, Boston, 1989.

[27] B. Jayaram. On the law of importation $(x \wedge$ $y) \rightarrow z \equiv(x \rightarrow(y \rightarrow z))$ in fuzzy logic. IEEE Transactions on Fuzzy Systems, 16:130144, 2008.

[28] L. Nosková. Systems of fuzzy relation equation with inf- $\rightarrow$ composition: solvability and solutions. J. of Electrical Engineering, 12(s):69-72, 2005.

[29] B. De Baets. A note on Mamdani controllers. In D. Ruan, P. D'hondt, P. Govaerts, and E. Kerre, editors, Intelligent Systems and Soft Computing for Nuclear Science and Industry (Proc. 2th FLINS), pages 22-28, Singapore, 1996. World Scientific. 